\title{
Frecuencia de atenciones por disfunción vestibular periférica en hospitales de nivel III, Perú
}

\author{
Frequency of medical evaluations for peripheral vestibular dysfunction in hospitals level III, Peru
}

Raquel Tapia-Egoavil 1,a, Marlene Luisa Cabrera-Iturrizaga ${ }^{1, b}$, Jorge López-Soria ${ }^{2, b}$

\section{RESUMEN}

La disfunción vestibular periférica produce un síndrome vestibular que compromete las actividades de la vida diaria lo cual genera discapacidad y un impacto negativo en la calidad de vida. Su prevalencia es alta y aumenta con la edad; hace sobreuso de recursos sanitarios por la alta tasa de recurrencia que eleva la carga de enfermedad. Objetivo: Establecer las frecuencias de atenciones por disfunción vestibular periférica según diagnóstico, edad, sexo, y atenciones por especialidad. Material y métodos: El estudio fue observacional y descriptivo, de las atenciones en los hospitales de nivel III del Ministerio de Salud (MINSA) a nivel nacional; la información fue obtenida de la base de datos del MINSA, en un periodo de tres años. Resultados: Con un total de 18340 atenciones, predominaron las atenciones del sexo femenino en $68 \%$, en todos los diagnósticos estudiados, las que fueron brindadas principalmente por otorrinolaringología y neurología. Las atenciones más frecuentes fueron el síndrome vertiginoso no especificado $46,10 \%$, otros vértigos periféricos $18,58 \%$ y vértigo posicional paroxístico benigno 16,91\%. Conclusiones: Las atenciones predominaron en mujeres, fueron realizadas principalmente por Otorrinolaringología y el diagnóstico más frecuente fue el síndrome vertiginoso no especificado. La información de la demanda de atenciones, permitirá planificar una respuesta sanitaria adecuada.

PALABRAS CLAVE: Enfermedades vestibulares, epidemiología, Perú. (Fuente: DeCS BIREME).

\section{SUMMARY}

Peripheral vestibular dysfunction produces a vestibular syndrome that affects routine daily activities leading to incapacity and impacting negatively on quality of life. The prevalence of this condition is high and increases with age, and it consumes significant resources due to the relapsing nature of the condition rising the burden of disease. Objective: To determine the frequency of medical evaluations for peripheral vestibular dysfunction by diagnosis, age, gender and by the specialty offering the medical evaluation. Methods: A descriptive and observational study was carried-out including hospitals level III ruled by the Ministry of Health (MOH) at a national scale, data were gathered from the MOH database over a period of 3 years. Results: A total of 18349 medical evaluations were analyzed; $68 \%$ of them occurred in females and were offered by neurologist and otorhinolaryngologysts. The most common diagnoses were non-specified vertiginous syndrome $46.10 \%$, other peripheral vertigo $18.58 \%$ and benign paroxysmal vertigo $16.91 \%$. Conclusions: Most of the medical evaluation occurred in women by

Departamento de Investigación, Docencia y Rehabilitación Integral en Amputados, Quemados y Trastornos Posturales, Instituto Nacional de Rehabilitación Dra. Adriana Rebaza Flores Amistad Perú - Japón. Chorrillos, Perú.

2 Departamento de Investigación, Docencia y Rehabilitación Integral en Lesiones Centrales, Instituto Nacional de Rehabilitación Dra. Adriana Rebaza Flores Amistad Perú - Japón. Chorrillos, Perú.

a Médico rehabilitador;

b Tecnólogo Médico en el área Terapia Física y Rehabilitación 
otorhinolaryngologysts, the most common diagnosis was non-specified vertiginous syndrome. This information may allow to plan an adequate sanitary response.

KEYWORDS: Vestibular diseases, epidemiology, Peru. (Source: MeSH NLM).

\section{INTRODUCCIÓN}

La disfunción vestibular periférica es una condición clínica que compromete el sistema vestibular y altera las respuestas posturales y oculomotoras, lo cual produce un síndrome vestibular (SV) que se caracteriza por vértigo, nistagmo, ataxia, signos neurovegetativos, lateropulsión $(1,2)$, inestabilidad postural $\mathrm{y}$ desdibujamiento visual al movimiento de la cabeza (3). Cuando la disfunción es bilateral, provoca visión borrosa al mover la cabeza (oscilopsia), desequilibrio crónico e inestabilidad postural discapacitante (4).

El SV tiene componentes estáticos (desviación oblicua, asimetrías posturales) y dinámicos (desequilibrio, oscilopsia) (5), que comprometen las actividades sociales, familiares y profesionales con perjuicios físicos, financieros y psicológicos (6).

Las causas pueden ser por lesiones periféricas (laberinto o nervio vestibular) o de las vías vestibulares centrales (generalmente del tronco del encéfalo o el cerebelo) (7). Las lesiones vestibulares consideradas en la Clasificación Internacional de Enfermedades (CIE10) son: enfermedad de Menière, vértigo posicional paroxístico benigno (VPPB), neuronitis vestibular, laberintitis, otros vértigos periféricos, otros trastornos de la función vestibular, síndrome vertiginoso no especificado, síndromes vertiginosos en enfermedades clasificadas en otra parte, laberintitis, disfunción del laberinto y disfunción vestibular central (8).

La prevalencia de disfunción vestibular en adultos es variable; $1,8 \%$ en Corea (9), 3,1\% en Taiwán (10), $7,4 \%$ en Alemania (11) y $35,4 \%$ en EEUU (12); esto podría ser atribuido al uso de protocolos diferentes o a poblaciones diferentes (9). Predomina en el sexo femenino y aumenta con la edad (10), la prevalencia en niños es de 5,3\% (13). La disfunción vestibular bilateral ocurre en 28 por 100000 adultos (4).

La disfunción vestibular produce discapacidad física relacionada a riesgo de caídas (12), interrupción de las actividades diarias, días laborales perdidos y abandono laboral $(14,15)$, que afectan la calidad de vida (11) y generan un impacto negativo personal, social y económico (14).
Se ha observado en otros países una alta tasa de recurrencia y reingreso de pacientes por disfunción vestibular $(10,16)$, por lo que es necesario conocer la demanda y el contexto en el que se brinda estas atenciones. En el Perú no existe estudios que cuantifiquen la frecuencia de atenciones por disfunción vestibular; por lo tanto disponer de información estadística general es importante para orientar la toma de decisiones en la atención y planificación de programas de intervención.

El objetivo de esta investigación fue establecer la frecuencia de atenciones por disfunción vestibular periférica según diagnóstico, edad, sexo, y atenciones por especialidad, en hospitales de nivel III del Ministerio de Salud del Perú.

\section{MATERIAL Y MÉTODOS}

Estudio observacional no experimental, transversal y descriptivo realizado en el Instituto Nacional de Rehabilitación (INR), entre marzo y noviembre del 2018. Se registraron las atenciones de los hospitales de nivel III del Ministerio de Salud (MINSA) a nivel nacional, en el periodo 2015-2017.

Los datos fueron obtenidos de la base de datos de la Oficina de Tecnologías de la Información del MINSA, según la codificación de la CIE-10 correspondiente a disfunción vestibular periférica. Se utilizó una hoja de registro para consignar la distribución por sexo, grupos de edad y atenciones por especialidad. Esta información fue procesada en MS Excel para su análisis.

El análisis de la información se realizó a toda la población atendida con códigos diagnósticos CIE-10 H81.0, H81.1, H81.2, H81.3, H81.8, H81.9, H82X, H83.0, H83.2 (disfunción vestibular periférica) y H81.4 (disfunción vestibular central).

La distribución por edades se describió en intervalos de 5 años desde 0 hasta 99 años de edad.

Se evaluó la significancia de la asociación entre sexo y diagnóstico mediante la prueba de Chi cuadrado considerando un $\mathrm{p}<0,05$ como significativo. 
El protocolo de investigación fue revisado y aprobado por los Comités de Ética e Investigación del INR.

\section{RESULTADOS}

En el periodo 2015-2017, se realizaron 18340 atenciones por disfunción vestibular periférica en los hospitales de nivel III del MINSA; 12465 (68\%) de las atenciones correspondieron al sexo femenino y $5875(32 \%)$ al sexo masculino.

Las atenciones más frecuentes según diagnósticos CIE-10 fueron el síndrome vertiginoso no especificado
$46,10 \%$, otros vértigos periféricos $18,58 \%$ y VPPB $16,91 \%$. La distribución de las atenciones por diagnósticos según sexo se muestra en la tabla 1.

Se encontró asociación significativa entre sexo y las atenciones por cada diagnóstico $(\mathrm{p}=0,02)$. En la proporción de varones y mujeres, la laberintitis y neuronitis vestibular presentaron una proporción de varones ligeramente mayor en comparación a los otros diagnósticos (34\% y 36\% respectivamente). No se encontró asociación significativa en las atenciones por disfunción vestibular central o periférica y el sexo $(\mathrm{p}=0,13)$ (tabla 1$)$.

Tabla 1. Frecuencia de atenciones por disfunción vestibular según sexo y diagnóstico.

\begin{tabular}{|c|c|c|c|c|}
\hline Diagnóstico & Varones (\% fila) & Mujeres (\% fila) & Total & $\mathbf{p}$ \\
\hline (H81.0) Enfermedad de Meniere & $309(32)$ & $652(68)$ & 961 & 0.02 \\
\hline (H81.1) Vértigo paroxístico benigno & $1005(32)$ & $2097(68)$ & 3102 & \\
\hline (H81.2) Neuronitis vestibular & $159(36)$ & $281(64)$ & 440 & \\
\hline (H81.3) otros vértigos periféricos & $1065(31)$ & $2342(69)$ & 3407 & \\
\hline (H81.8) Otros trastornos de la función vestibular & $95(32)$ & $198(68)$ & 293 & \\
\hline (H81.9) Síndrome vertiginoso no especificado & $2646(31)$ & $5809(69)$ & 8455 & \\
\hline $\begin{array}{l}\text { (H82X) Sindromes vertiginosos en enfermedades } \\
\text { clasificadas en otra parte }\end{array}$ & $177(34)$ & $349(66)$ & 526 & \\
\hline (H83.0) Laberintitis & $395(37)$ & $683(63)$ & 1078 & \\
\hline (H83.2) Disfunción del laberinto & $24(31)$ & $54(69)$ & 78 & \\
\hline Total, disfunciones periféricas & $5875(32)$ & $12465(68)$ & 18340 & 0.13 \\
\hline Total, disfunciones centrales & $183(35)$ & $336(65)$ & 519 & \\
\hline
\end{tabular}

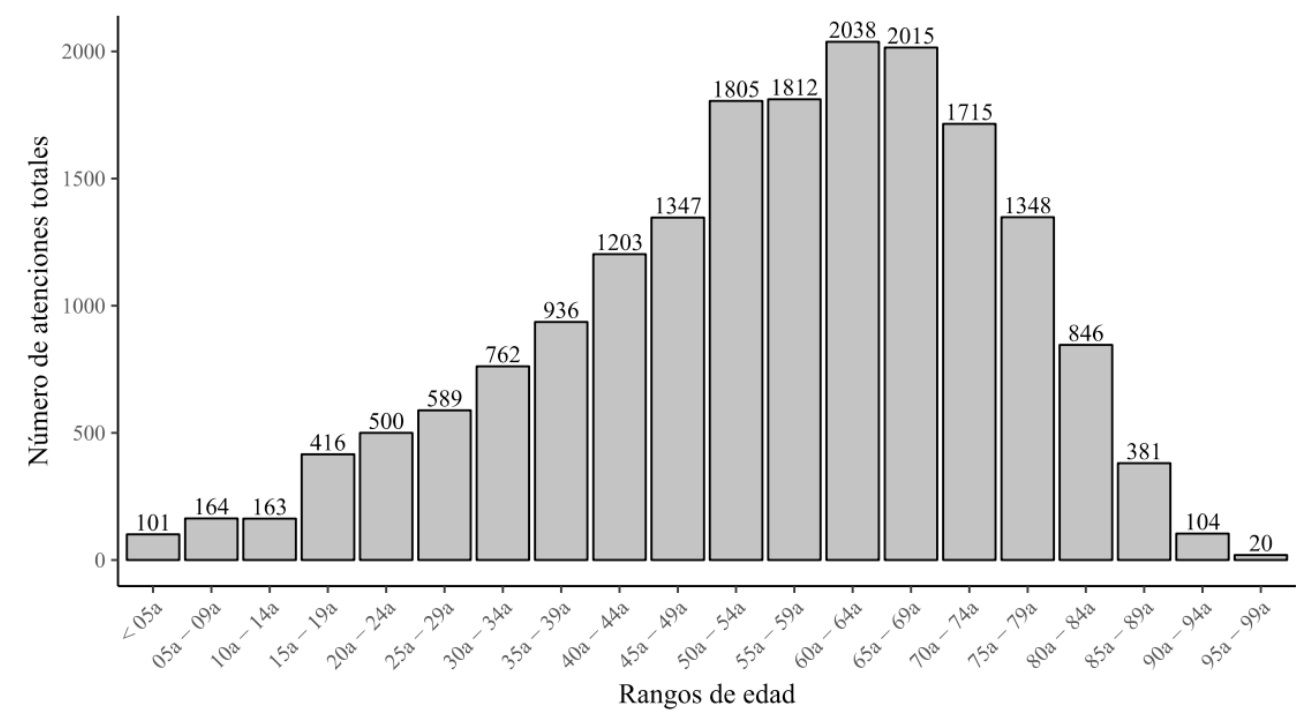

Gráfico 1. Frecuencia de atenciones por disfunción vestibular periférica según grupo etario. 
Tabla 2. Distribución de las atenciones por disfunciones vestibulares periféricas según grupo etario.

\begin{tabular}{lcccccccccc}
\hline Edad & H81.0 & H81.1 & H81.2 & H81.3 & H81.8 & H81.9 & H82X & H83.2 & H83.0 & Total \\
\hline$<05 \mathrm{a}$ & 2 & 16 & 7 & 8 & 5 & 32 & 2 & 3 & 26 & 101 \\
$05 \mathrm{a}-09 \mathrm{a}$ & 3 & 17 & 12 & 20 & 8 & 50 & 2 & 3 & 49 & 164 \\
$10 \mathrm{a}-14 \mathrm{a}$ & 3 & 25 & 3 & 27 & 3 & 55 & 5 & 3 & 39 & 163 \\
$15 \mathrm{a}-19 \mathrm{a}$ & 17 & 62 & 13 & 67 & 6 & 188 & 11 & 2 & 50 & 416 \\
$20 \mathrm{a}-24 \mathrm{a}$ & 24 & 78 & 18 & 84 & 5 & 241 & 11 & 4 & 35 & 500 \\
$25 \mathrm{a}-29 \mathrm{a}$ & 37 & 100 & 12 & 84 & 7 & 278 & 20 & 7 & 44 & 589 \\
$30 \mathrm{a}-34 \mathrm{a}$ & 48 & 128 & 24 & 132 & 14 & 340 & 26 & 4 & 46 & 762 \\
$35 \mathrm{a}-39 \mathrm{a}$ & 61 & 170 & 20 & 163 & 13 & 420 & 29 & 3 & 57 & 936 \\
$40 \mathrm{a}-44 \mathrm{a}$ & 74 & 187 & 34 & 216 & 17 & 580 & 26 & 9 & 60 & 1203 \\
$45 \mathrm{a}-49 \mathrm{a}$ & 79 & 251 & 28 & 227 & 11 & 619 & 38 & 5 & 89 & 1347 \\
$50 \mathrm{a}-54 \mathrm{a}$ & 106 & 286 & 54 & 322 & 26 & 834 & 40 & 10 & 127 & 1805 \\
$55 \mathrm{a}-59 \mathrm{a}$ & 84 & 308 & 39 & 344 & 28 & 826 & 69 & 9 & 105 & 1812 \\
$60 \mathrm{a}-64 \mathrm{a}$ & 104 & 347 & 51 & 405 & 35 & 928 & 60 & 5 & 103 & 2038 \\
$65 \mathrm{a}-69 \mathrm{a}$ & 98 & 347 & 44 & 396 & 32 & 936 & 57 & 1 & 104 & 2015 \\
$70 \mathrm{a}-74 \mathrm{a}$ & 104 & 291 & 31 & 332 & 28 & 813 & 46 & 4 & 66 & 1715 \\
$75 \mathrm{a}-79 \mathrm{a}$ & 62 & 244 & 23 & 268 & 21 & 643 & 48 & 1 & 38 & 1348 \\
$80 \mathrm{a}-84 \mathrm{a}$ & 38 & 147 & 22 & 155 & 22 & 407 & 29 & 1 & 25 & 846 \\
$85 \mathrm{a}-89 \mathrm{a}$ & 15 & 64 & 3 & 68 & 7 & 204 & 6 & 1 & 13 & 381 \\
$90 \mathrm{a}-94 \mathrm{a}$ & 2 & 29 & 2 & 18 & 5 & 47 & 0 & 0 & 1 & 104 \\
$95 \mathrm{a}-99 \mathrm{a}$ & 0 & 4 & 0 & 6 & 0 & 8 & 1 & 0 & 1 & 20 \\
\hline
\end{tabular}

Nota: H81.0=Enfermedad de Meniere, H81.1=Vértigo paroxístico benigno, H81.2=Neuronitis vestibular, H81.3=Otros vértigos periféricos, $\mathrm{H} 81.8=$ Otros trastornos de la función vestibular, H81.9=Síndrome vertiginoso no especificado, H82X=Síndromes vertiginosos en enfermedades clasificadas en otra parte, H83.0=Laberintitis, H83.2=Disfunción del laberinto

Tabla 3. Distribución de atenciones por disfunciones vestibulares periféricas según especialidad.

\begin{tabular}{|c|c|c|c|c|c|c|c|c|}
\hline Diagnóstico & OTL & Neurología & Pediatría & Geriatría & $\begin{array}{l}\text { Medicina } \\
\text { interna }\end{array}$ & $\begin{array}{l}\text { Medicina } \\
\text { general }\end{array}$ & Otros & $\begin{array}{l}\text { No } \\
\text { informada }\end{array}$ \\
\hline Enfermedad de Menière & 420 & 282 & 2 & 22 & 37 & 34 & 38 & 32 \\
\hline Vértigo Paroxístico Benigno & 1522 & 594 & 9 & 105 & 185 & 57 & 120 & 418 \\
\hline Neuronitis Vestibular & 278 & 37 & 0 & 2 & 4 & 3 & 21 & 17 \\
\hline Otros Vértigos Periféricos & 1585 & 765 & 9 & 171 & 227 & 126 & 171 & 79 \\
\hline $\begin{array}{l}\text { Otros Trastornos de la } \\
\text { Función Vestibular }\end{array}$ & 133 & 45 & 0 & 2 & 8 & 4 & 63 & 25 \\
\hline $\begin{array}{l}\text { Trastorno de la Función } \\
\text { Vestibular, no Especificado }\end{array}$ & 3907 & 1252 & 42 & 369 & 1119 & 1077 & 440 & 20 \\
\hline $\begin{array}{l}\text { Síndromes Vertiginosos } \\
\text { Clasificados en otra parte }\end{array}$ & 129 & 36 & 4 & 9 & 53 & 27 & 32 & 176 \\
\hline Laberintitis & 802 & 18 & 10 & 2 & 64 & 61 & 75 & 3 \\
\hline Disfunción del Laberinto & 49 & 0 & 0 & 0 & 1 & 1 & 5 & 19 \\
\hline TOTAL & $\begin{array}{l}8825 \\
(50,6 \%)\end{array}$ & $\begin{array}{l}3029 \\
(17,4 \%)\end{array}$ & $76(0,4 \%)$ & $\begin{array}{l}682 \\
(3,9 \%)\end{array}$ & $\begin{array}{l}1698 \\
(9,7 \%)\end{array}$ & $\begin{array}{l}1390 \\
(8,0 \%)\end{array}$ & $\begin{array}{l}965 \\
(5,5 \%)\end{array}$ & $789(4,5 \%)$ \\
\hline
\end{tabular}


En relación a los grupos etarios, la mayor frecuencia de atenciones fue en el grupo de 60 a 64 años con 2038 atenciones y en el grupo de 64 a 69 años con 2015 atenciones (gráfico 1).

En cada uno de los grupos etarios mayores de 15 años, el diagnóstico especificado con más atenciones fue el VPPB. Por el contrario, en los grupos etarios menores de 15 años las atenciones fueron predominantemente por laberintitis (tabla 2).

Las atenciones fueron realizadas principalmente por la especialidad de Otorrinolaringología 50,6\% y Neurología $17,4 \%$ del total (tabla 3 ).

\section{DISCUSIÓN}

En el presente estudio se analizó la información estadística proporcionada por el Ministerio de Salud de Perú, que reportó un total de 18340 atenciones por disfunción vestibular periférica realizadas desde el año 2015 al 2017.

En relación a la distribución por sexo, existe predominio femenino en un $68 \%$ de las atenciones en la población estudiada. Lopez-Gentili et al. (17), y Reis et al. (18), informaron el 63,1\% y $68,3 \%$ de disfunción vestibular en mujeres, respectivamente, si bien son investigaciones realizadas en pacientes atendidos, se ve una clara preponderancia en el sexo femenino.

Cuando se analizó cada uno de los diagnósticos CIE-10, en todos ellos hubo predominio femenino, pero se observó que la laberintitis y neuronitis vestibular presentaron una proporción de varones ligeramente mayor en comparación a los otros diagnósticos (34\% y $36 \%$, respectivamente), sin embargo, estos valores presentan desviaciones mínimas.

En algunas investigaciones sobre VPPB también se reportan una tasa más alta en mujeres, como el de Messina et al. (19), con el 60,9\% y el estudio de prevalencia poblacional de VPPB realizada por Von Brevern et al. (20), que informaron 3,2\% en mujeres frente a $1,6 \%$ en hombres. En nuestro estudio, aunque corresponden a número de atenciones, también se evidenció el predominio del sexo femenino $67,6 \%$ de las atenciones por VPPB.

En la distribución de las atenciones según edad, se observó una alta frecuencia en el rango de 50 a 74 años. Si bien la frecuencia de atenciones aumenta con la edad, se observa un descenso a partir de los 70 años, lo que podría estar relacionado con la disminución de la tasa poblacional general y no con un descenso de la prevalencia de disfunciones vestibulares periféricas. De hecho, está demostrado en estudios poblacionales que la prevalencia de vértigo aumenta con la edad $(9,10,12)$.

Es importante mencionar que en nuestra población, el diagnóstico más frecuentemente informado correspondió al síndrome vertiginoso no especificado, $46,1 \%$ del total de atenciones por disfunción vestibular periférica. En un estudio, realizado en a una población anciana, se señaló que el 19,3\% de los casos su causa era desconocida (21). Esto pone de relieve que, debido a factores como la ambigüedad del cuadro clínico en su etapa inicial, la necesidad de una evaluación otoneurológica muy especializada y la falta de equipamiento para pruebas vestibulares, en un gran porcentaje de pacientes que presentan disfunciones vestibulares no se puede precisar un diagnóstico definitivo.

En este estudio, el VPPB fue el diagnóstico especificado con mayor frecuencia de atenciones. Otros estudios sobre vértigo también muestran que el VPPB es la causa más frecuente de consulta (16) y la causa más común en ancianos (21). Asimismo, en todos los grupos etarios, el VPPB fue el diagnóstico especificado con mayor frecuencia de atenciones, a excepción de los menores de 15 años en quienes las atenciones más frecuentes fueron por laberintitis.

La mayoría de atenciones fueron realizadas por la especialidad de Otorrinolaringología 50,6\% seguido de Neurología 17,4\%. Un estudio que utilizó la base de datos de casos atendidos, reportó también que la mayoría fueron atendidos por otorrinolaringólogos (10).

La frecuencia de atenciones presentadas puede ser por consultas reiteradas, característica descrita en pacientes que padecen disfunción vestibular. Así, Lai et al. (10), reportan una alta tasa de recurrencia $37,7 \%$ y von Brevern et al. (20), informan reingreso de $78 \%$ por el mismo síntoma. En el VPPB también existe una recurrencia de $52,5 \%$ a $55 \%(19,20)$.

Esta demanda de atenciones genera mayor uso de recursos sanitarios, como lo muestra el estudio en emergencia hospitalaria de otorrinolaringología, donde el $10,8 \%$ de atendidos corresponde a trastornos del equilibrio y vértigo (18). 
Con fines de comparación, se muestra el número de atenciones por disfunción vestibular central 2,8\% frente a la disfunción vestibular periférica $97,2 \%$ del total de atenciones. Se han realizado estudios en pacientes atendidos que señalan diferentes proporciones al comparar disfunción vestibular central y periférica, como el de Uruguay, donde del $71 \%$ que correspondía a un vértigo verdadero, $48 \%$ fue periférico y $23 \%$ central (16) y el de Brasil que reporta $77 \%$ periférico y $23 \%$ central (22). En nuestro estudio el bajo porcentaje de la disfunción vestibular central podría estar en relación a subdiagnóstico o estar reportados con otros códigos CIE-10, como la migraña vestibular que pertenecen a una disfunción vestibular central.

Este trabajo tiene varias limitaciones. En primer lugar, el análisis retrospectivo de registros de la atención médica puede ser una fuente de sesgo. En segundo lugar, la precisión de la codificación de los diagnósticos por los profesionales médicos puede afectar la validez de los datos. En tercer lugar, la información proporcionada por el MINSA tiene errores porque no coinciden las sumatorias según los reportes por sexo comparadas por edad y por especialidad, además no cuenta con la información de las atenciones de cuatro hospitales del año 2017 y hubo datos faltantes en la información relacionada a la atención por especialidad. Sin embargo, la fortaleza del presente estudio es la población a nivel nacional, con un gran tamaño de la muestra, considerándose representativa de las atenciones en los hospitales de Nivel III del MINSA, además por ser referencia de los otros niveles de atención, la mayor parte de las atenciones son brindadas por médicos especialistas.

El abordaje de la disfunción vestibular periférica incluye tratamiento médico y rehabilitación vestibular (RV). La RV es efectiva porque controla el SV $(23,24)$, comprende aspectos físicos, emocionales y funcionales; logra mejorar el equilibrio, la independencia, la calidad de vida y el nivel de actividad del paciente, además de disminuir las caídas $(5,25,26)$, condiciones que facilitan su reincorporación laboral y participación en la sociedad, las que disminuye los costos tanto de los pacientes con SV como las del país (24).

En conclusión, en este estudio hubo predominio del sexo femenino, las atenciones fueron realizadas principalmente por la especialidad de otorrinolaringología y el diagnóstico más frecuente fue el síndrome vertiginoso no especificado. La información de la demanda de atenciones, permitirá planificar una respuesta sanitaria adecuada.
Es necesario realizar investigaciones de prevalencia y factores de riesgo para precisar la carga de enfermedad, con la finalidad de tomar decisiones y diseñar programas de intervención.

\section{Institución donde se realizó el estudio:}

Instituto Nacional de Rehabilitación Dra. Adriana Rebaza Flores Amistad Perú-Japón

\section{Declaración de financiamiento y de conflictos de interés:}

El estudio fue financiado por el Instituto Nacional de Rehabilitación Adriana Rebaza Flores Amistad PerúJapón. Los autores declaran no tener conflictos de intereses

\section{Contribución de autoría:}

RTE; MLCI y JLS: Participaron en el diseño del estudio, recolección de los datos, interpretación de los resultados y aprobación de la versión final a ser publicada.

\section{Correspondencia:}

Raquel Tapia-Egoavil

Correo electrónico: raqueltapiaego@gmail.com

\section{REFERENCIAS BIBLIOGRÁFICAS}

1. Fustinoni O. Semiología del sistema nervioso de Fustinoni. 14. ${ }^{a}$ ed. El Ateneo; 2006. p. 517.

2. Sauvage JP. Vertigos: manual de diagnóstico y rehabilitación. 2. ${ }^{a}$ ed. Barcelona: Elsevier; 2017.

3. Hall CD, Herdman SJ, Whitney SL, et al. Vestibular rehabilitation for peripheral vestibular hypofunction: An evidence-based clinical practice guideline. J Neurol Phys Ther. 2016; 40(2):124-55.

4. Ward BK, Agrawal Y, Hoffman HJ, Carey JP, Della Santina CC. Prevalence and impact of bilateral vestibular hypofunction: Results from the 2008 United States National Health Interview Survey. JAMAOtolaryngolHead Neck Surg. 2013;139(8):80310.

5. Herdman SJ. Vestibular Rehabilitation. 3. ${ }^{a}$ ed. Filadelfia: FA Davis Company; 2007.

6. Castro ASO de, Gazzola JM, Natour J, Ganança FF. Brazilian version of the dizziness handicap inventory. Pró-Fono Rev Atualização Científica. 2007;19(1):97104.

7. Kasper D, Fauci A, Hauser S, Longo D, Jameson JL, Loscalzo J. Harrison. Principios de medicina interna. 
Madrid: McGraw-HIll; 2016.

8. Organización Panamericana de la Salud. Clasificación estadística internacional de enfermedades y problemas relacionados con la salud. $10 .^{\mathrm{a}}$ ed. Washington D.C: Organización Panamericana de la Salud; 1995.

9. Koo JW, Chang MY, Woo SY, Kim S, Cho YS. Prevalence of vestibular dysfunction and associated factors in South Korea., Prevalence of vestibular dysfunction and associated factors in South Korea. BMJ Open BMJ Open. 2015; 5:e008224-e008224.

10. Lai Y-T, Wang T-C, Chuang L-J, Chen M-H, Wang P-C. Epidemiology of vertigo: a National Survey. Otolaryngol Neck Surg. 2011; 145(1):110-6.

11. Neuhauser HK, Radtke A, Von MB, Lezius F, Feldmann M, Lempert T. Burden of dizziness and vertigo in the community. Arch Intern Med. 2008; 168(19):2118-24.

12. Agrawal Y, Carey JP, Della Santina CC, Schubert MC, Minor LB. Disorders of Balance and Vestibular Function in US Adults: Data From the National Health and Nutrition Examination Survey, 20012004. Arch Intern Med. 2009; 169(10):938.

13. Li C-M, Hoffman HJ, Ward BK, Cohen HS, Rine RM. Epidemiology of dizziness and balance problems in children in the United States: A population-based study. J Pediatr. 2016; 171:240-247.

14. Benecke H, Agus S, Kuessner D, Goodall G, Strupp M. The Burden and Impact of Vertigo: Findings from the REVERT Patient Registry. Front Neurol. 2013; 4: 136. doi: 10.3389/fneur.2013.00136

15. Gutiérrez-Márquez A, Jáuregui-Renaud K, ViverosRenteria L, Villanueva-Padrón LA. Discapacidad por enfermedad auditiva y vestibular en un centro de atención especializada. Gac Médica México. 2005; 141(2):105-10.

16. Castro L, Braga P. Mareo y/o vértigo como motivo de consulta en la policlínica neurológica: estudio descriptivo. Rev Médica Urug. 2018; 29(4):208-218.
17. López-Gentili LI, Kremenchutzky M, Salgado P. A statistical analysis of 1300 patients with dizzinessvertigo. Its most frequent causes. Rev Neurol. 2003; 36(5):417-20.

18. Reis LR, Lameiras R, Cavilhas P, Escada P. Epidemiology of Vertigo on Hospital Emergency. Acta Médica Port. 2016; 29(5):326-31.

19. Messina A, Casani AP, Manfrin M, Guidetti G. Italian survey on benign paroxysmal positional vertigo. Acta Otorhinolaryngol Ital. 2017; 37(4):328-35.

20. von Brevern M, Radtke A, Lezius F, et al. Epidemiology of benign paroxysmal positional vertigo: a population-based study. J Neurol Neurosurg Psychiatry. 2007; 78(7):710-5.

21. Sogebi OA, Ariba AJ, Otulana TO, Osalusi BS. Vestibular disorders in elderly patients: characteristics, causes and consequences. Pan Afr Med J. 2014; 19:146.

22. Mostafa BE, Kahky AO, Kader HM, et al. Central vestibular dysfunction in an otorhinolaryngological vestibular unit: Incidence and diagnostic strategy. Int Arch Otorhinolaryngol. 2014; 18(3):235-8.

23. Arnold S, Stewart A, Moor H, Karl R, Reneker J. The effectiveness of vestibular rehabilitation interventions in treating unilateral peripheral vestibular disorders: A Systematic Review. Physiother Res Int. 2017 Jul; 22(3). doi: 10.1002/pri.1635

24. Miranda M, Santana J, Fernandez CA. Síndrome vertiginoso periférico: programa individualizado de ejercicios físicos para su rehabilitación. Rev Arch Méd Camagüey. 2010; 14(3):0-0.

25. Júnior R, Roberto $\mathrm{P}$, Kozan ES, et al. Vestibular rehabilitation in the quality of life and the symptomatology of dizziness among the elderly. Ciênc Amp Saúde Coletiva. 2014; 19(8):3365-74.

26. Peres M, Silveira E da. Effect of vestibular rehabilitation in elder people: regarding balance, quality of life and perception. Ciênc Amp Saúde Coletiva. 2010; 15(6):2805-14.

Recibido: 16/01/2019

Aceptado: 28/03/2019 